\title{
Span Morphing Using the Compliant Spar
}

\author{
R. M. Ajaj ${ }^{1}$; E. I. Saavedra Flores ${ }^{2}$; M. I. Friswell ${ }^{3}$; and F. A. Diaz De la $\mathrm{O}^{4}$
}

\begin{abstract}
This paper develops and models the compliant spar concept that allows the wingspan to be varied to provide roll control and enhance the operational performance for a medium altitude, long endurance (MALE) UAV. The wing semispan is split into morphing partitions and the concept may be incorporated in each partition; however, only the tip partition is considered here. The compliant spar is made of compliant joints arranged in series to allow the partition to be flexible under axial (spanwise) loads, but at the same time stiff enough to resist bending loads. Each compliant joint consists of two concentric overlapping AL 2024-T3 tubes joined together using elastomeric material. Under axial (spanwise) loading, the elastomeric material deforms in shear, allowing the overlapping distance between the tubes to vary and hence the length (in the spanwise direction) of the joint/spar to vary. High fidelity modeling of the concept is performed. Then, structural design optimization studies are performed to minimize the axial stiffness and the structural mass of the concept for various design constraints. The flexible skin and actuation system to be used are also addressed. DOI: 10.1061/(ASCE)AS.1943-5525.0000442. @ 2014 American Society of Civil Engineers.
\end{abstract}

\section{Introduction}

Continuous demands to enhance flight performance and control authority have focused the interest of aircraft designers on span morphing. Wings with large spans have good range and fuel efficiency, but lack maneuverability and have relatively low cruise speeds. By contrast, aircraft with low aspect ratio wings can fly faster and become more maneuverable, but show poor aerodynamic efficiency (McCormik 1995). A variable span wing can potentially integrate into a single aircraft the advantages of both designs, making this emerging technology especially attractive for military unmanned aerial vehicle (UAVs). Increasing the wingspanincreases the aspect ratio and wing area and decreases the spanwise lift distribution for the same lift. Thus, the drag of the wing could be decreased, and consequently, the range or endurance of the vehicle increased. Unfortunately, the wing-root bending moment can increase considerably due to the larger span. Thus the aerodynamic, structural, aeroelastic, and control characteristics of the vehicle should be investigated in the design of variable-span morphing wings. Most span morphing concepts are based on a telescopic mechanism, following the ideas of Ivan Makhonine, a Russian expatriate. With this mechanism, the wing's outer panel telescoped inside the inner panel to enable span and wing area changes. The MAK-10 was the first design with a telescopic wing and it first flew in 1931. The mechanism was powered pneumatically and enabled span increases up to $62 \%$ (from 13 to $21 \mathrm{~m}$ ) and area increases up to $57 \%$ (from 21 to $33 \mathrm{~m}^{2}$ ) (Weisshaar 2006). Blondeau et al. (2007) designed and fabricated a three-segmented telescopic wing

${ }^{1}$ Lecturer in Aerospace Structures, Aeronautics and Astronautics, Univ. of Southampton, Southampton SO17 1BJ, U.K. (corresponding author). E-mail:r.ajaj@southampton.ac.uk

${ }^{2}$ Assistant Professor, Departamento de Ingeniería en Obras Civiles, Universidad de Santiago de Chile, Ave. Ecuador 3659, Santiago, Chile.

${ }^{3}$ Professor of Aerospace Structures, College of Engineering, Swansea Univ., Swansea SA2 8PP, U.K.

${ }^{4}$ Lecturer, School of Engineering, Univ. of Liverpool, Liverpool L69 3GQ, U.K.

Note. This manuscript was submitted on November 12, 2013; approved on June 9, 2014; published online on August 11, 2014. Discussion period open until January 11, 2015; separate discussions must be submitted for individual papers. This paper is part of the Journal of Aerospace Engineering, (C) ASCE, ISSN 0893-1321/04014108(13)/\$25.00. for a UAV. Hollow fiberglass shells were used to preserve the spanwise airfoil geometry and ensure compact storage and deployment of the telescopic wing. To reduce the weight, they replaced the wing spars with inflatable actuators that could support the aerodynamic loads on the wing (in excess of $73 \mathrm{~kg} / \mathrm{m}^{2}$ ). Their telescopic spar design consisted of three concentric circular aluminum tubes of decreasing diameter and increasing length, connected by ceramic linear bearings, and it was deployed and retracted using input pressures of $345-483 \mathrm{kPa}(50-70 \mathrm{psi})$. The wing could undergo a $114 \%$ change in the aspect ratio, while supporting aerodynamic loads.

Blondeau et al. (2003) adopted two identical telescopic spars instead of one, mechanically coupled by the ribs, to prevent wing twist and fluttering. The new prototype could undergo a $230 \%$ change in aspect ratio, and seam heights were reduced to give less parasitic drag. In its fully deployed condition, the telescopic wing could achieve lift-to-drag ratios as high as 16 , which was similar to its solid foam-core wing counterpart. The most dramatic morphing wing involving span change that has been realized as a wind tunnel prototype is the Agile Hunter by Lockheed Martin (Ivanco et al. 2007; Bye and McClure 2007; Love et al. 2007). Funded by defense advanced research projects agency (DARPA) within the morphing aircraft structures (MAS) program, the prototype was based on a military UAV capable of folding the inner sections of the wing near the fuselage to reduce the surface area and drag during transonic flight at low altitude (also called a Z-wing). The major challenge was the realization of suitable hinges that connect the two wing portions; the hinges have to sustain the aerodynamic loads but offer a smooth, continuous aerodynamic surface. Several materials were considered, including silicone-based and shape memory polymer skins. Wind tunnel tests at Mach 0.6 showed a morphing capability from $0^{\circ}$ to $130^{\circ}$ over $65 \mathrm{~s}$ with a controllable, reliable, and precise actuation. Bae et al. (2005) performed both static aerodynamic and aeroelastic studies on the wing of a longrange cruise missile and highlighted some of the benefits and challenges associated with the design of a morphing wing capable of span change. The total drag decreased by approximately $25 \%$, and the range increased by approximately $30 \%$. The aeroelastic analysis showed that the flexibility of the morphing wing structure increased as the wingspan increased. At a given flight condition, the deformation from the aerodynamic loads was much larger than that of the conventional wing. Static aeroelastic considerations that 


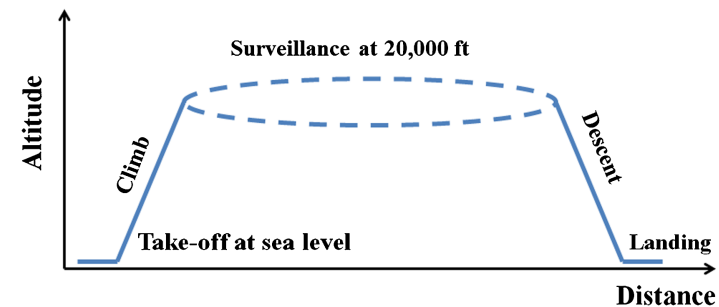

Fig. 1. Mission profile of the UAV

a variable-span wing requires increased bending stiffness because the bending deformation is more significant than twist. Ajaj et al. (2012a, 2013) developed the Zigzag wingbox concept that allows the wingspan of a medium altitude, long endurance (MALE) UAV to be varied by $44 \%$ ( $22 \%$ extension and $22 \%$ retraction). The Zigzag wingbox consists of a rigid part and a morphing part. The morphing part consists of various morphing partitions where in each partition there are two spars, each consisting of two beams hinged together. Each morphing partition is covered by flexible skin and is bounded by two ribs through which the spars are connected. Equivalent modeling and preliminary sizing of the concept are performed to assess its feasibility and quantify its potential benefits.

Asymmetrical span morphing can be used for roll control. Seigler et al. (2004) investigated asymmetrical span extension for increased maneuverability of bank-to-turn cruise missiles. By formulating a full nonlinear model of the missile, due to the shift of the missile's center of mass and the dependence of the rolling moment on the angle of attack, they showed that the control authority can be significantly larger when compared to conventional tail surface control. Improved maneuverability, however, is highly dependent upon the angle of attack, linear actuation speed, and extension length. Moreover, as the mass of the extending wings becomes large relative to the missile body, the rigid body dynamics can become increasingly complex and a nonlinear control law was formulated to control the roll, angle of attack, and sideslip angle dynamics in accordance with bank-to-turn guidance. The control method proved to be adept in tracking commanded inputs while effectively eliminating sideslip. Seigler et al. (2007) studied the modeling and flight control of vehicles with large-scale planform changes. They derived equations of atmospheric flight in a general form, examined methods to integrate the aerodynamic forces, and distinguished among various approaches and methods of flight control. A more extensive review on span morphing technology (applications and concepts) for both fixed-wing and rotary-wing aircraft is given in Barbarino et al. (2011).

The main objective of this paper is to develop a structural concept that allows the wingspan to be varied to provide roll control and enhance the operational performance of a MALE UAV. High fidelity modeling and structural optimization studies are performed to maximize the ratio of bending to axial stiffness and minimize the structural mass of the concept. The flexible skin and actuation system to be used are also addressed.

\section{Benefits of Span Morphing}

Ajaj et al. (2012b, 2014) investigated the use of asymmetric span morphing to provide roll control and replace conventional ailerons. In addition, they assessed the potential benefits of symmetric span morphing in reducing vortex-induced drag, extending endurance, and reducing takeoff field length (TOFL) and landing distance (LD) for a MALE UAV. The UAV they considered is similar to the BAE Systems Herti UAV (Austin 2010). The UAV has a maximum lift-to-drag ratio of about 20 and a maximum endurance capability of about $18 \mathrm{~h}$. A representative flight profile, shown in Fig. 1, was assumed in this analysis. The UAV takes off with a weight of $800 \mathrm{~kg}$, cruises and loiters for about $18 \mathrm{~h}$ with a speed of $50 \mathrm{~m} / \mathrm{s}$ (M0.16) at 6,100 $\mathrm{m}(20,000 \mathrm{ft})$, and then descends and lands.

The design parameters of the vehicle are given in Table 1.

Ajaj et al. (2012b, 2014) used the Tornado vortex lattice method (VLM) to estimate induced drag. Parasitic drag (skin friction and form) were estimated using semiempirical correlations. Endurance is expected to vary as the accuracy of the aerodynamic model increases, but the variation will be small as endurance is mainly related to the loitering flight phase at which the angle of attack is fairly small, and the flow is expected to remain attached. The Tornado vortex lattice method predicts induced drag with a high level of accuracy for low angles of attack, which is the case in this paper. However, the profile drag estimation is based on semiempirical correlations that are reasonably accurate with an error margin. If a higher fidelity aerodynamic model is used, the estimated endurance will increase slightly, as the semiempirical correlations tend to overestimate the profile drag. A convergence study was performed to ensure that the prediction of their VLM code is robust with minimal numerical errors. The outcomes of their study can be summarized as follows (Ajaj et al. 2012b, 2014):

- The wing must be able to extend on both sides by up to $22 \%$ and must be able to retract on both sides by up to $22 \%$ to provide sufficient roll control over the entire flight profile;

- The rolling moment generated by asymmetric span morphing is very sensitive to the angle of attack (AOA). This sensitivity to AOA does not exist with conventional ailerons. This proves that morphing structures should not be operated in the same way as conventional control surfaces. The benefits that can be achieved from coupled maneuvers must be exploited via the design of ad hoc flight control systems;

- Span morphing induces some additional inertial terms in the roll equation of motion. These increase the importance of the transient response compared to ailerons due to the larger and heavier structure to be actuated;

- Assuming the baseline basic operating weight (BOW), a $6.5 \%$ increase in endurance can be achieved at $35 \%$ span extension. However, at $22 \%$ span extension, a $6 \%$ increase in endurance can be achieved, and from structural and actuation perspectives $22 \%$ is more feasible and easier to manufacture and implement; and

- Finally, the wing designed to extend and retract up to $22 \%$ can achieve a $28 \%$ reduction in TOFL and a $10 \%$ reduction in LD.

The endurance is referred to as the time spent airborne during loiter without including the time spent during takeoff and landing. However, the amount of fuel needed during takeoff and landing is considered. The sensitivity of the result to weight is considered, while the impacts of additional cost and manufacturing complexity

Table 1. Design Parameters of the UAV

\begin{tabular}{lc}
\hline Design parameters & Values \\
\hline MTOW & $800 \mathrm{~kg}$ \\
BOW & $500 \mathrm{~kg}$ \\
Fuel weight & $150 \mathrm{~kg}$ \\
Endurance & $18 \mathrm{~h}$ \\
Lift-to-drag & 20 \\
Airfoil & NACA $63-015 \mathrm{~A}$ \\
Span & $12 \mathrm{~m}$ \\
Chord & $1.87 \mathrm{~m}$ \\
Wing area & $22.44 \mathrm{~m}^{2}$ \\
\hline
\end{tabular}

Note: $\mathrm{BOW}=$ basic operating weight; MTOW = maximum takeoff weight. 


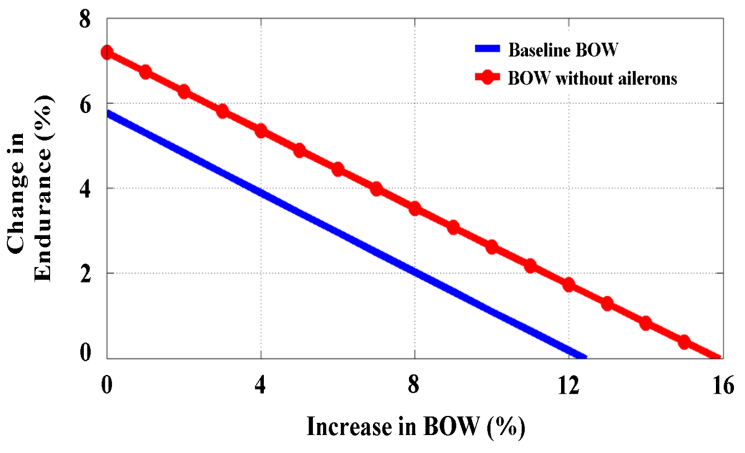

Fig. 2. Change of endurance with the BOW at $22 \%$ span extension

are not considered. This resulted in $22 \%$ span morphing (extension and retraction) being a good compromise, capable of delivering sufficient roll control (the same as the baseline aileron), while extending flight endurance by $6 \%$ and enhancing the takeoff and landing phases. Therefore, the compliant spar is designed to be capable of extending by $22 \%$ and retracting by $22 \%$ on each side of the wing.

It should be noted that the ultimate benefits of the span morphing technology decrease as the BOW increases. The baseline BOW includes the weight of the conventional ailerons which will be removed when the span morphing technology is incorporated. The structural weight of the ailerons with their internal supports is estimated to be $15 \mathrm{~kg}$, using modified semiempirical correlations from Torenbeek (1992). The variations of endurance with baseline BOW and BOW without the ailerons at $22 \%$ symmetric span extension are shown in Fig. 2.

The variation of endurance with BOW is not linear, but in the region of up to a $16 \%$ increase in $\mathrm{BOW}$, it can be approximated as linear. The potential increase in endurance that can be achieved with a $22 \%$ symmetric span extension decreases as the BOW increases. In fact, the endurance can go even lower than that of the baseline UAV if the BOW of the morphing vehicle exceeds that of the baseline vehicle by more than $12.5 \%$ (for the baseline BOW) and $16 \%$ (for the BOW without ailerons). This implies that the span morphing system (structure and actuator) must be as light as possible to maximize the gain in endurance.

In the literature, there are many span morphing concepts, varying from telescopic structures with sliding skins actuated pneumatically or hydraulically to compliant structures with flexible skins actuated using shape memory alloy (SMA) or muscles. However, only a limited number of aircraft with span morphing wings have been built and flown, as shown in Fig. 3. There are many reasons for this and they are beyond the scope of this paper. Table 2 summarizes the morphing concepts (structure and actuation) used with those aircraft.

Table 2 shows that most of the vehicles used telescopic structures in which the morphing partition(s) can slide in and out

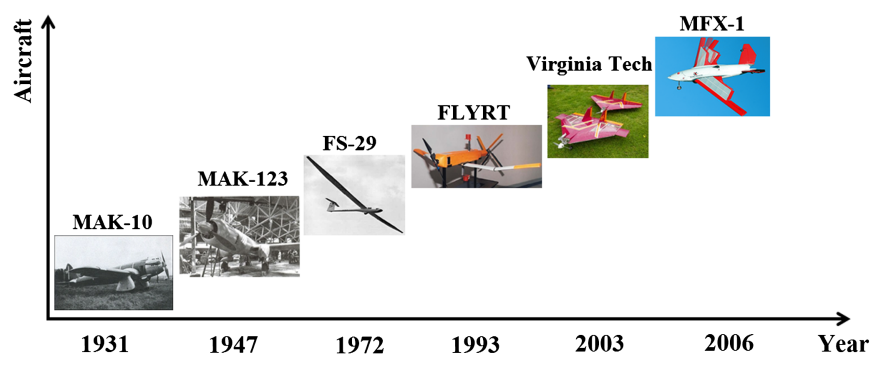

Fig. 3. Aircraft that flew span morphing wings (image by Rafic Ajaj) through the fixed inboard partition. These vehicles do not require any compliant or flexible skin, as the sliding/telescopic mechanism allows rigid covers and semimonocoque construction. This is the main driver for developing the compliant spar concept, to enable a fully compliant structure that allows the coupling of span and sweep and distributed actuation along the span of the wing.

\section{Compliant Spar}

The compliant spar concept consists of compliant joints that allow the spar to be flexible under axial (spanwise) loads but at the same time stiff enough to resist bending loads. The compliant joint (Element 2) shown in Fig. 4(a) consists of two concentric overlapping AL 2024-T3 tubes joined together using elastomeric material. Under axial (spanwise) loading, the elastomeric material deforms in shear, allowing the overlapping distance between the tubes to vary and hence the length (in the spanwise direction) of the joint/spar to vary. The elastomeric material is bonded to the $\mathrm{AL}$ tubes. The bonding process and the manufacturing/integration of the spar are beyond the scope of this paper.

To assess the feasibility of the compliant spar concept, the wing semispan is split into five morphing partitions and the compliant spar is embedded in each partition. In this paper, only the wingtip morphing partition is studied. The entire wing with compliant spars will be considered in future work. The wingtip partition has two compliant spars bounded by two major ribs, one at the root and one at the tip. The front spar is located at $20 \%$ of the chord while the rear spar is located at $65 \%$ of the chord. A schematic of the compliant spar concept inside the tip morphing partition is shown in Fig. 4(b).

Low fidelity structural modeling was avoided as the intention is to maximize the efficiency of the elastomer usage by varying its distribution along the length and the cross section of the joint to maximize the ratio of bending to axial stiffness. This is difficult to capture accurately enough with low fidelity modeling. High fidelity modeling of the structure is performed using ANSYS 13. The term high fidelity modeling is used to emphasize the high level of geometric detail that the structural model presents. This type of modeling differs from lower fidelity structural models, which focus on the overall description of the physical system rather than capturing detailed information of the mechanics and physics of each of the components that constitute the global system. Low fidelity structural models are normally based on analytical calculations instead of using (generally time consuming) numerical procedures, and they are particularly preferred for conceptual studies and preliminary sizing of components at much more reduced computing times. The detailed geometric information that the structural model presents is an intermediate step to reach much higher levels of fidelity. These expensive models may include nonlinear geometric effects, nonlinear material properties (particularly important to capture the constitutive response of the elastomeric material), aeroelastic coupling, etc. In fact, with the adoption of much higher levels of fidelity, the computing times and memory requirements soon would become prohibitive, making this alternative unfeasible with the current computational technology available at present. Therefore, as an intermediate step in this modeling process, it is found to be more convenient (and justifiable) to ignore nonlinear effects and aeroelastic coupling in the analyses.

The aerodynamic loads (bending moment and shear force) are extracted from the Tornado VLM. Since the wing consists of different morphing partitions, there are various ways to achieve this. In other words, either all of the partitions extend or retract by the same amount or they extend or retract by different amounts to achieve an overall span change of $22 \%$. The main focus here is on the first 
Table 2. Air Vehicles Flown with Span Morphing Technology

\begin{tabular}{lcccc}
\hline Vehicle & Category & Morphing & Structure & Skin \\
\hline MAK-10 & GAA & Span & Telescopic & Sliding \\
MAK-123 & GAA & Span & Telescopic & Sliding \\
FS-20 & Glider & Span & Telescopic & Sliding \\
FLYRT & UAV & Span & Pneumatic & Screw jacks \\
Virginia tech & UAV & Span & Telescopic & Sliding \\
MFX-1 & UAV & Aspect ratio and sweep & Articulated lattice structure & Stretchable \\
\hline
\end{tabular}

Note: GAA = general aviation aircraft.

scenario where all of the morphing partitions are extended or retracted by the same amount, which is $22 \%$. This implies that the wingtip partition, studied here, has to be able to extend or retract by $22 \%$ of its original length.

\section{Flexible Skin and Minor Ribs}

The two spars are covered by flexible skin to allow the partition length to be varied. The compliant spar concept requires a skin surface that can extend along with the underlying structure while effectively carrying the aerodynamic pressure loads with minimal out-of-plane deformations. The skin therefore needs to have large in-plane strain capability with low stiffness and simultaneously high out-of-plane stiffness.

The flexible skin adopted here is a sandwich panel consisting of tensioned elastomeric matrix composite (EMC) covers reinforced by a zero Poisson's ratio cellular core. The EMCs consist of either a silicone or polyurethane elastomer matrix reinforced with carbon fibers (Bubert et al. 2010; Murray et al. 2010). These fibers are aligned nearly perpendicular to the spanwise direction, so that they do not add significantly to the overall spanwise bending skin stiffness, but do transfer loads in the chordwise direction.

Additionally, they effectively eliminate the otherwise large Poisson's ratio of the elastomer matrix. The elastomeric covers are pretensioned, because increasing the amount of tension in the skin increases its out-of-plane stiffness without increasing weight. The zero Poisson's ratio cellular core is the MorphCore developed by Kothera et al. (U.S. Patent No. 7,931,240) specifically for
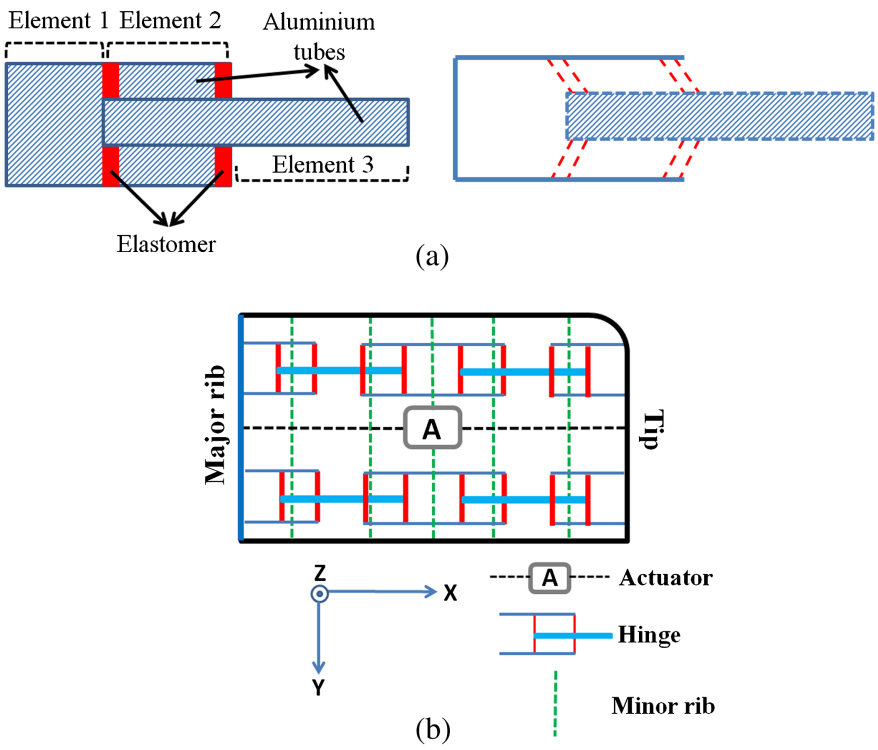

Fig. 4. Compliant spar concept: (a) the compliant joint (before and after actuation); (b) the wingtip morphing partition morphing wing applications. The geometry of the core creates two distinct structural components. The points of the chevrons serve as bending beams, whose high aspect ratio and small angle relative to the chordwise direction create a spanwise extensional stiffness. The sides of the chevrons are all aligned, creating continuous ribs. These ribs provide a continuous surface for bonding the EMC skin and give the MorphCore an effective Poisson's ratio of zero. The close spacing of the ribs and bending beams provides distributed support for the EMC skin, reducing its unsupported length and significantly decreasing out-of-plane deflections.

In order to maintain the aerodynamic shape of the airfoil and prevent excessive deformation of the skin, minor ribs are installed between the major ribs. The flexible skin is simply supported at the major and minor ribs to prevent excessive deformation of the skin under aerodynamic loads that can jeopardize the aerodynamic benefits of span morphing.

The minor ribs are attached only to Elements 1 or 2 through the holes in the ribs, as shown in Fig. 5(a). Minor ribs cannot be attached to Elements 3 as this constrains the ability of the spar/ partition to retract. It should be noted that the compliant spar always starts and ends with Element 1, the large diameter AL tube. Elements 1 attached to the root major rib are stationary and hence minor ribs attached to those elements are also stationary. In contrast, Element 1 attached to the tip major rib moves with the rib and not relative to it. Minor ribs attached to Elements 1 (other than those attached to major ribs) and Elements 2 move relative to the root major rib as shown in Figs. 5(b and c). The deformation (stretching) of the flexible skin during extension is shown in Fig. 5(c). The flexible skin is pretensioned to maintain the aerodynamic shape of the airfoil when the partition is retracted by $22 \%$.

\section{Structural Modeling in ANSYS}

The concentric tubes, made from AL 2024-T3, are modeled using the SHELL181 element in ANSYS. SHELL181 is suitable for analyzing thin to moderately thick shell structures. It has four nodes with six degrees of freedom per node. Furthermore, it is suitable for linear large rotation, and both full and reduced integration schemes are supported (ANSYS 13). The elastomer is modeled using the SOLID185 element. The SOLID185 is used for three-dimensional modeling of solid structures. It has eight nodes, each node having three degrees of freedom (translations in the nodal $x, y$, and $z$ directions). The element formulation allows deformations of nearly incompressible elastoplastic materials and fully incompressible hyperelastic materials to be simulated (ANSYS 13). A rigid beam element is used to model the major rib at one side of the partition where the other side is clamped. The rigid beam is modeled using the MPC184 element which is well suited for linear, large rotation applications. MPC184 is a multipoint constraint element that applies kinematic constraints between nodes (Groover 2010). The major rib ensures that the front and rear spar work in parallel in resisting the out-of-plane and in-plane loads. 


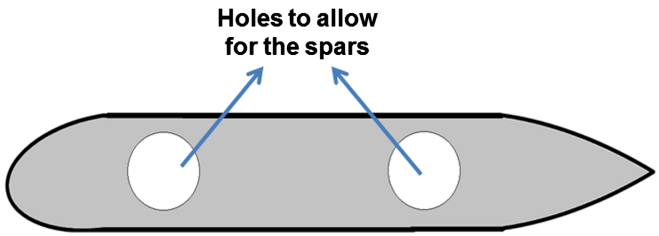

(a)

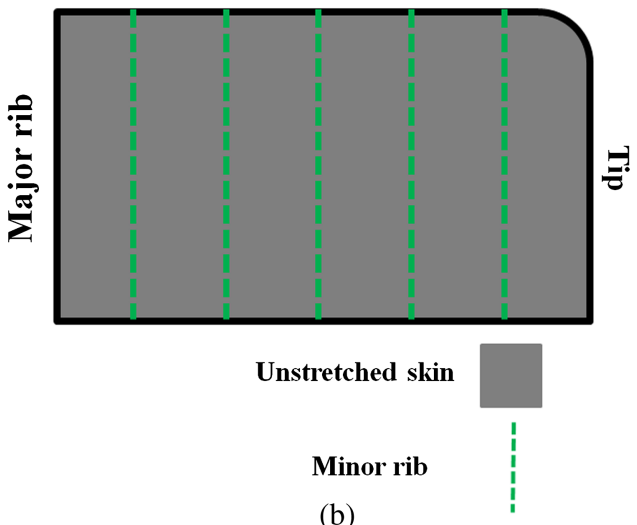

(b)

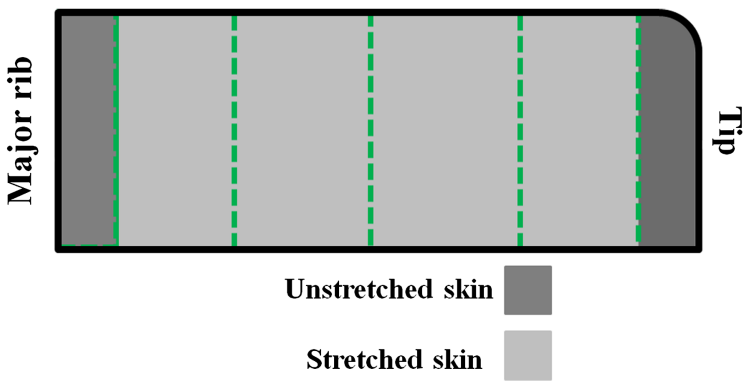

(c)

Fig. 5. Minor ribs to support the flexible skin: (a) schematic of a minor rib; (b) partition before extension; (c) partition after extension

A convergence study of the mesh size was carried out in order to minimize numerical errors (such as spurious volumetric locking, i.e., overstiff solutions) associated with the size of each finite element. The study consisted of testing several finite element meshes of different geometries of a single compliant joint belonging to the full spar. To produce the meshes, the automatic mesh generator implemented in ANSYS is adopted, which allows the user to specify the maximum mesh size (that is, the maximum size of each finite element), while maintaining mesh connectivity automatically. As the optimization study was computationally expensive, the optimal size is carefully chosen, trying to find a balance between the desired accuracy and reasonable computation times. A typical optimal finite element mesh consisted of 7,650 SHELL181 elements, 960 SOLID185 three-dimensional elements, and 25,697 nodes. When compared to the analysis of a much finer mesh consisting of 21,440 SHELL181 elements, 5,760 SOLID185 elements, and 126,051 nodes, the analysis of the optimal mesh took approximately $27 \%$ of the time spent in the analysis of the finer model. In addition, the tip deflection computed with the optimal model was just $1.3 \%$ larger than that obtained in the much finer model. The analysis in this paper is based on the following assumptions:

- The strain in the elastomer due to span extension is below the value at which the behavior of the elastomer becomes nonlinear;

- Nonlinear geometric effects are neglected; and
Table 3. Material Properties of Polyurethane Elastomer (Data from Groover 2010)

\begin{tabular}{lc}
\hline Properties & Value \\
\hline$E$ & $10 \mathrm{MPa}$ \\
$\sigma_{\text {allow }}$ & $45 \mathrm{MPa}$ \\
$\rho$ & $1,250 \mathrm{~kg} / \mathrm{m}^{3}$ \\
$v$ & 0.5 \\
\hline
\end{tabular}

- The elastomer is assumed to be isotropic and fully incompressible.

The material and geometric nonlinearity assumptions are necessary to avoid the analysis from becoming virtually prohibitive in terms of computing times. In addition, the fully incompressible isotropic material law is a widely accepted assumption in the modeling of elastomeric materials and represents a reasonable description (Groover 2010).

As highlighted before, the compliant joint consists of two concentric overlapping AL 2024-T3 tubes joined together using elastomeric material. The design of such a compliant joint is quite challenging. The bending stiffness must be high while the axial stiffness (spanwise direction) must be very low to minimize the actuation requirements. In addition, the weight of the partition must be low to maintain the benefits of the span morphing technology. Polyurethane elastomer is used in this analysis and the material properties are given in Table 3 .

In order to achieve low axial stiffness while maintaining high bending stiffness, the elastomer must be distributed in an efficient way to achieve maximum utilization of the material. Various configurations in the compliant joint are considered to distribute the elastomer, as shown in Fig. 6.

A qualitative approach was used to determine the most suitable configuration and cross section to be adopted. In Configuration 1, the elastomer is continuous along the length of the joint/Element 2. The weight and axial stiffness associated with this configuration are very high and the bending stiffness is also high. In fact, the elastomeric region near the ends of the joint works most efficiently to resist the bending loads. Therefore, in Configuration 2 the

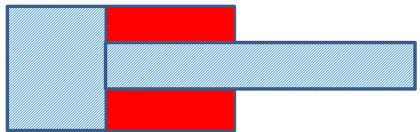

(a)

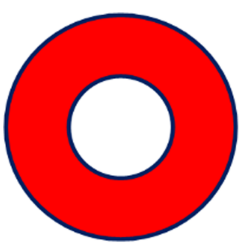

(c)

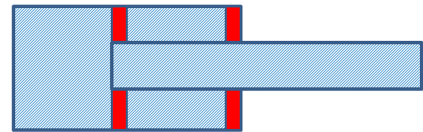

(b)

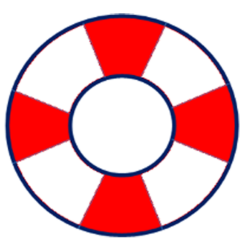

(d)

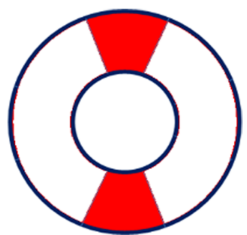

(e)

Fig. 6. Different joint configurations and elastomer cross sections considered: (a) Configuration 1; (b) Configuration 2; (c) Cross section 1; (d) Cross section 2; (e) Cross section 3 
elastomer is placed only near the two ends of joint/Element 2 to maximize the efficiency of the material in resisting the bending loads. This also reduces structural mass and increases the ratio of bending to axial stiffness. Configuration 2 is incorporated in this analysis as it is more promising in providing a high ratio of bending to axial stiffness with low structural mass.

Furthermore, three cross sections of the elastomeric material are considered, as shown in Figs. 6(c-e). Cross Sections 1 and 2 are more stable and provide better redundancy when compared to Cross Section 3, but the material usage to resist the bending and shear loads due to lift is not optimized, resulting in lower structural efficiency, suboptimal structural mass, and large actuation force. In Cross Section 2, the elastomers on the sides (left and right) work mainly in resisting the loads due to drag which are about 20 times smaller than the lift. The sides' elastomers contribute a little in resisting the loads due to lift. By removing those elastomers on the sides, Cross Section 3 is obtained. In Cross Section 3, the elastomers (top and bottom) work mainly in resisting loads due to lift and therefore maximizing the efficiency of the material usage. In addition, Cross Section 3 increases the ratio of bending to axial stiffness (lower actuation forces). Therefore, Configuration 2 with Cross Section 3 is adopted for the compliant joints. The choice of Cross Section 3 allows reduced computational time since a very fine mesh is required to capture the large deformations of the rubber.

Fig. 7 shows the compliant spars of the wingtip partition labeled and meshed in ANSYS 13. The distance separating the centers of the spars is $0.85 \mathrm{~m}$. Each spar consists of six joints (which is the general case). The spars are clamped on one side (root of the partition), and on the other side (tip of the partition) they are constrained together to artificially model the effect of the major rib at the tip.

It should be noted that the representative compliant spars shown in Fig. 8 are not the optimized version of the concept that has minimal axial stiffness and sufficient bending stiffness. The deformations of those spars under $1 g$ aerodynamic loads and $22 \%$ span extension in different views are shown in Fig. 8.

\section{Loads: Aerodynamics and Actuation}

The Tornado VLM was used for aerodynamic predictions. Tornado VLM is a linear aerodynamics code, and discounts wing thickness and viscous effects. These limitations imply that Tornado can only be used for angles of attack up to $8-10^{\circ}$ for slender wings (Melin $2000 \mathrm{a}, \mathrm{b})$. The aerodynamic forces and moments are extracted from Tornado VLM. These forces and moments are used to estimate an

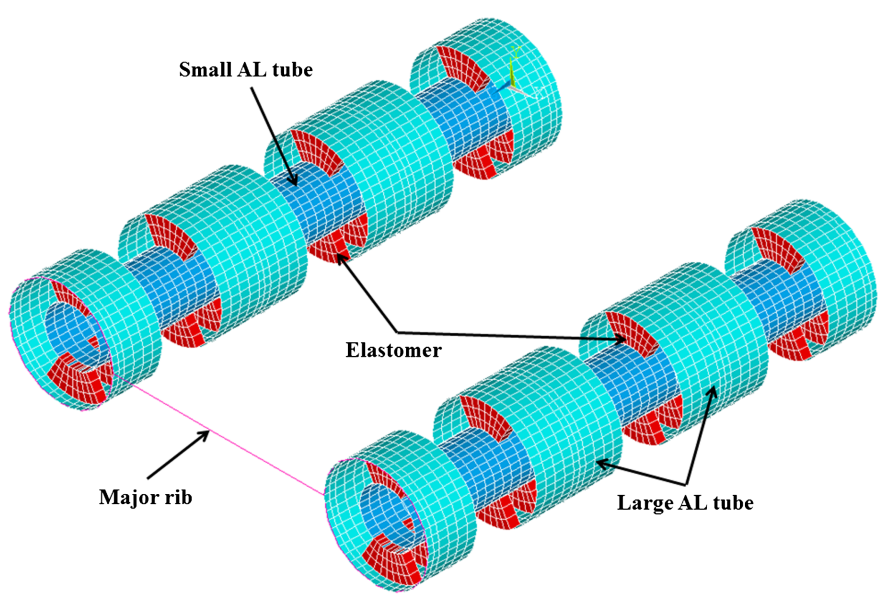

Fig. 7. Compliant spars in ANSYS 13 equivalent shear force and equivalent torque that are placed at the end of the partition (tip) on the midpoint of the connecting element/ major rib. The UAV can experience a load factor up to $6 g$ due to vertical gusts (Neubauer et al. 2007). This implies that the tip morphing partition (mainly the elastomer) must withstand those high limit loads without excessive deformations and/or permanent plastic deformation, especially if the span morphing is used to provide various functionalities, including roll control, which is essential for the safety and survivability of the UAV. Therefore, the aerodynamic loads extracted from Tornado VLM are amplified to simulate the $6 g$ scenario. It should be noted that at $6 g$, the geometry of the spar will be distorted but it should not fail permanently. The distortion of the spar (wing) reduces the aerodynamic efficiency, but this can be useful at this scenario to provide passive relief by dumping part of the life providing relief for the internal structure.

In addition to the aerodynamic loads, an axial (spanwise) extension equivalent to $22 \%$ of the partition length is imposed at the end of the partition to simulate the $22 \%$ extension scenario.

Finally, the self-weight of the partition is also considered in the analysis. This provides some inertial relief for the aerodynamic loads. The worst case loading scenario, full extension at $6 g$ vertical gust, is considered in this paper with a safety factor (SF) of 1.5 according to FAR Part 23 Section 303.

\section{Structural Optimization Studies}

Two structural optimization studies are performed in this section. In the first study, the axial stiffness of the partition is minimized for different geometric constraints. In the second study, the optimum solutions obtained from minimizing the axial stiffness are further optimized to minimize the structural mass of the partition. These studies are performed using a structural optimization suite that consists of the genetic algorithim (GA) optimizer, Tornado VLM, and ANSYS 13. The GA optimizer is selected because it is capable of finding a global optimum and it is suitable for multiobjective

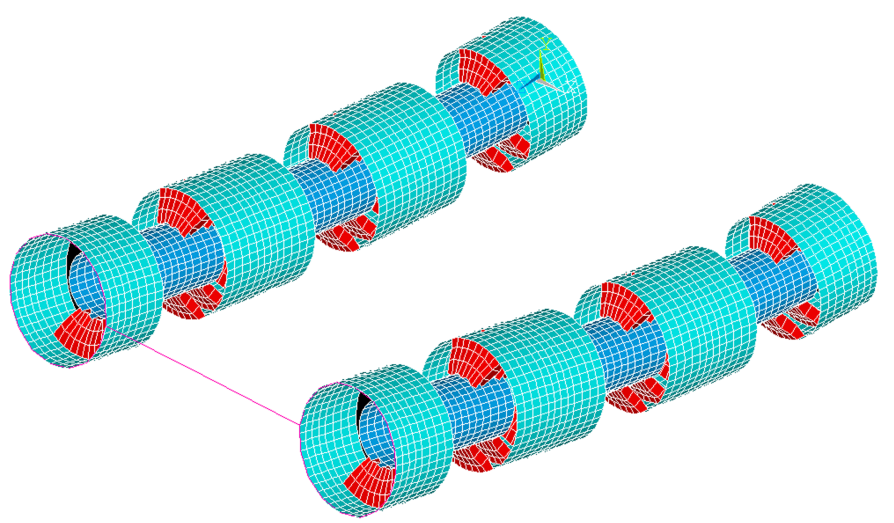

(a)

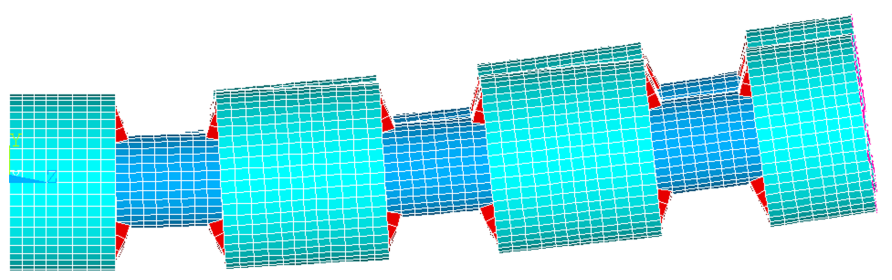

(b)

Fig. 8. Deformed compliant spars in various views: (a) isometric view; (b) side view 


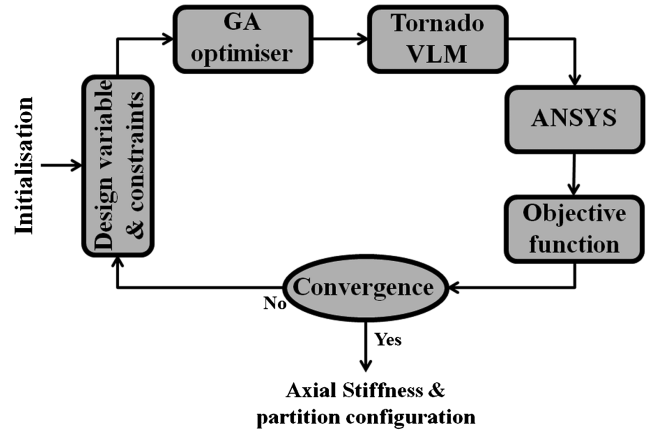

Fig. 9. Flowchart for the suite

optimization, which is the next analysis stage of the concept. Furthermore, GA optimizers are good for noisy environment and nonlinear problems and it is simple and easy to run in parallel, saving significant computational time and effort. A flowchart describing the optimization suite is shown in Fig. 9. It should be noted that aeroelastic coupling is not considered.

The Matlab GA Toolbox, developed by Chipperfield et al. (1994) and Chipperfield and Fleming (1996), was incorporated in this analysis. A fitness value is assigned to every individual of the initial population through an objective function that assesses the performance of the individual in the problem domain. Then, individuals are selected based on their fitness index and crossover between them is performed to generate new offspring. Finally, mutation of the new offspring is performed to ensure that the probability of searching any subspace of the problem is never zero. These abovementioned processes iterate until the optimum solution is achieved, depending on the convergence criteria of the problem. The optimization process has two constraint checks. The first check occurs after the GA has generated the design variables. If the combination of variables does not meet the constraints in the first check, then they will be disregarded (not passed to ANSYS) and a new set of variables will be generated. If the new design variables pass the first check, ANSYS is run, the results are extracted, and the second check is performed. If the results pass the check, then the objective function is assessed; else the individual will be given a predefined low value for its objective function.

\section{Axial Stiffness}

Reducing the axial stiffness of the compliant spars is a main design driver as it determines the magnitude of the actuation forces and power. The actuation forces and power have a direct impact on the size, weight, and number of the actuators required. The partition original length $\left(l_{p}\right)$ before morphing is $1.2 \mathrm{~m}$ (20\% of the wing semispan). A schematic showing the design variables of the compliant joint is shown in Fig. 10.

The objective is to minimize the axial stiffness of the partition, and hence the actuation force required, while maintaining sufficient bending stiffness $\left(K_{z}\right)$. The bending stiffness of the partition is incorporated as a constraint on the tip deflection at the $6 g$ gust load factor relative to the partition length.

Two different diameters of Element 1 are considered, given as $d_{1}=13.5 \%$ and $d_{1}=15 \%$ of the chord $(c=1.87)$. Two values of $d_{1}$ are considered here to account for two potential airfoils that have maximum thickness-to-chord ratios of 13.5 and $15 \%$. This is required to solve the optimization problem for the two values of $d_{1}$. These correspond to 0.2525 and $0.2805 \mathrm{~m}$, respectively. It should be noted that the value of $d_{1}$ is a constraint and not a variable in the optimization studies.

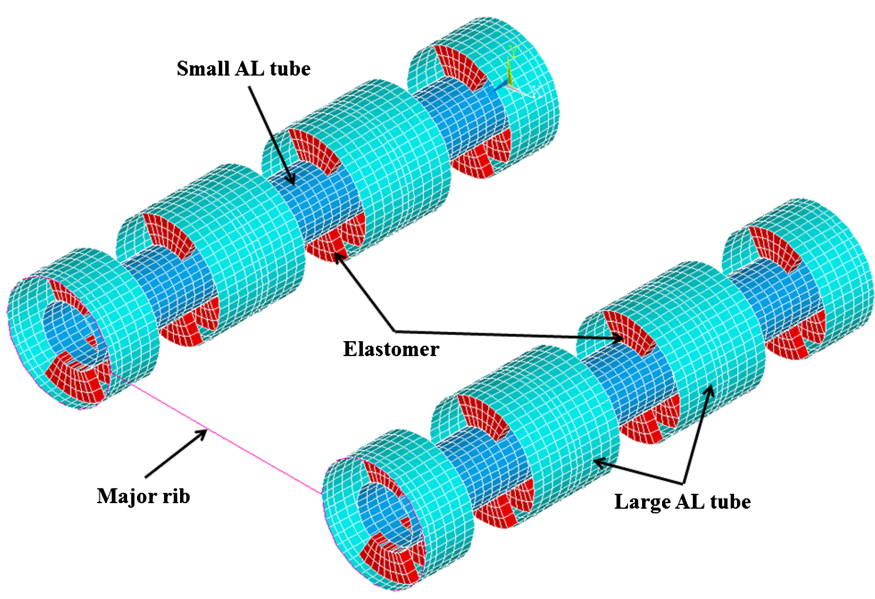

(a)

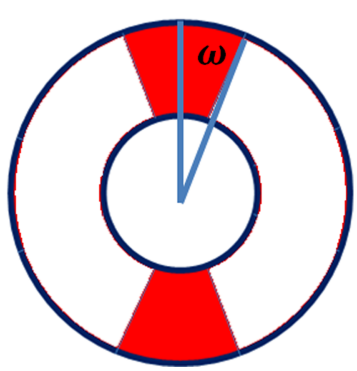

(b)

Fig. 10. Geometric parameters of a compliant joint

Since this analysis is linear, it is essential to consider a constraint on the aspect ratio of the elastomer to account for buckling and other nonlinear structural deformations that exist but are not captured in the linear analysis and can impact the stability of the concept. As the thickness of the elastomer (length $l_{e}$ in the axial direction) may decrease excessively during the optimization process (since the objective function is to minimize the axial stiffness), the slenderness of the elastomer may increase considerably and therefore, under high levels of compressive loads, buckling can occur. The elastomer may undergo a large amount of compression as bending loads are transferred between the two concentric tubes joined by the elastomeric material. In order to prevent the phenomenon of buckling in the system, three possible cases on the aspect ratio of the elastomers are considered. These cases establish the condition such that the elastomeric material reaches its ultimate compressive strain $\varepsilon^{\text {ult }}$ before buckling. This condition can be expressed mathematically as

$$
\varepsilon_{c r}=\frac{\pi^{2} I}{A(K L)^{2}}=\frac{\pi^{2}}{12}\left(\frac{2 l_{e}}{d_{1}-d_{3}}\right)^{2} \leq \varepsilon^{\mathrm{ult}}
$$

where $\varepsilon_{c r}=$ Euler or critical buckling strain; $I=$ moment of inertia; $K L=$ effective length of buckling; and $A=$ area of the elastomer which resists the compressive load. This simple formula leads to the condition

$$
\frac{2 l_{e}}{d_{1}-d_{3}} \leq \frac{1}{\pi} \sqrt{12 \varepsilon^{\mathrm{ult}}}
$$

which is used to calculate the constraining values shown in Table 4 of the paper. It should be noted that in this condition, the elastomer is considered to be fully fixed at one end and restrained to rotate at the other end, but allowing for lateral displacement. The first case 
Table 4. Cases for Elastomer Aspect Ratio

\begin{tabular}{lc}
\hline Constraint & Value \\
\hline Case 1 & $2 l_{e} /\left(d_{1}-d_{3}\right) \geq 0.0$ \\
Case 2 & $2 l_{e} /\left(d_{1}-d_{3}\right) \geq 0.1$ \\
Case 3 & $2 l_{e} /\left(d_{1}-d_{3}\right) \geq 0.2$ \\
\hline
\end{tabular}

(Case 1) considers no restriction on the elastomer's aspect ratio. This is represented by an ultimate strain $\varepsilon^{\text {ult }}$ equal to zero. The second and third cases (Cases 2 and 3, respectively) consider a maximum value of 0.01 and 0.03 for $\varepsilon^{\text {ult }}$, respectively. The choice of these values leads to the constraining values of 0.1 and 0.2 , respectively, as shown in Table 4 . Table 4 lists the three cases related to the aspect ratio of the elastomer.

In Case 1, there is no actual constraint on the minimum value elastomer's length of $l_{e}$, while in Case $2, l_{e}$ must be greater than or equal to $10 \%$ of the elastomer's radius. Finally in Case $3, l_{e}$ must be greater than or equal to $20 \%$ of the elastomer's radius.

A convergence study was undertaken to ensure using a fine mesh capable of capturing the sensitivity of the axial/bending stiffness to the elastomer thickness and angle. The optimization problem is run for the different values of $d_{1}$ separately. Table 5 summarizes the optimization problem with $d_{1}=0.135 \mathrm{c}$. In addition to the constraints listed in Table 5, the cases listed in Table 4 are considered separately and the optimization problem is repeated for each of them.

In Table $5, K_{x}$ is the axial stiffness of the partition, $n_{j}$ is the number of joints per spar, $l_{1_{\text {tot }}}$ is the total length of Elements 1, $l_{2_{\text {tot }}}$ is the total length of Elements $2, l_{3_{\mathrm{tot}}}$ is the total length of Elements 3 where $l_{p}=l_{1_{\text {tot }}}+l_{2_{\text {tot }}}+l_{3_{\text {tot }}}, d_{3}$ is the outer diameter of Element 3,c is the chord of the wing, $t_{1}$ is the thickness of the large AL tube, $t_{3}$ is the thickness of the small AL tube, $\omega$ is

Table 5. Optimization of the Axial Stiffness with Tight Bending Constraint

\begin{tabular}{lcc}
\hline Objective function & Minimize $K_{x}$ \\
\hline Variables & $n_{j}, l_{1_{\mathrm{tot}}}, l_{2_{\mathrm{tot}}}, l_{e}, d_{3}, t_{1}, t_{3}, \omega, \gamma$ \\
Constraints & $2 \leq n_{j} \leq 6$ & $0.03 c \leq d_{3} \leq 0.08 c$ \\
& $0.05 l_{p} \leq l_{1_{\mathrm{tot}}} \leq 0.25 l_{p}$ & $0.10 \leq t_{1} \leq 2.50\left(\times 10^{-3} \mathrm{~m}\right)$ \\
$0.30 l_{p} \leq l_{2_{\mathrm{tot}}} \leq .65 l_{p}$ & $1.00 \leq t_{3} \leq 3.00\left(\times 10^{-3} \mathrm{~m}\right)$ \\
$0.025 l_{2} \leq l_{e} \leq 0.25 l_{2}$ & $25^{\circ} \leq \omega \leq 75^{\circ}$ \\
$l_{1} \geq \delta x_{j}$ & $\mathrm{RF}_{1} \geq 1$ \\
$l_{3} \geq 2 \delta x_{j}$ & $\mathrm{RF}_{2} \geq 1$ \\
& $\delta z \leq 0.5 l_{p}$ & $\mathrm{RF}_{3} \geq 1$ \\
& $d_{1}=0.135 c$ & -
\end{tabular}

half of the angle bounding one elastomer section as shown in Fig. 10, $\mathrm{RF}_{1}$ is the reserve factor of the of the large $\mathrm{AL}$ tube, $\mathrm{RF}_{2}$ is the reserve factor of the elastomer material, $\mathrm{RF}_{3}$ is the reserve factor of the small AL tube, $\delta x_{j}$ is the axial deformation of the compliant joint, and $\delta z$ is the out-of-plane deformation of the tip end of the morphing partition. $l_{1}, l_{2}, l_{3}$, and $\delta x_{j}$ can be obtained from Eqs. (3)-(6), respectively, as

$$
\begin{gathered}
l_{1}=\frac{l_{1_{\mathrm{tot}}}}{0.5 n_{j}+1} \\
l_{2}=\frac{l_{2_{\mathrm{tot}}}}{n_{j}} \\
l_{3}=\frac{l_{p}-l_{1_{\mathrm{tot}}}-l_{2_{\mathrm{tot}}}}{0.5 n_{j}} \\
\delta x_{j}=\frac{0.22 l_{p}}{n_{j}}
\end{gathered}
$$

The RF constraints are essential to ensuring that the ultimate stresses in the AL tubes and the elastomer are below the corresponding allowable strengths. In addition to the RF constraints, two geometric constraints are added to ensure that when the partition is retracted by $22 \%$, the outer tubes (Elements 1 ) of the adjacent joints do not collide with each other.

After investigating the convergence criteria of the GA, the number of generations of the optimizer is fixed to 100, with 100 individuals per generation. For the sake of accuracy and consistency, each optimization run is repeated five times. Table 6 summarizes the outcomes of the study.

$l_{3_{\text {tot }}}=l_{p}-l_{1_{\text {tot }}}-l_{2_{\text {tot }}}$ and $m_{p}$ is the structural mass of partition. It should be noted that the GA optimizer reduces the angle $\omega$ toward the lower limit of the variable $\left(25^{\circ}\right)$ for both $d_{1}=0.135 \mathrm{c}$ and $d_{1}=$ $0.15 c$ at the different constraints. In addition, due to the large bending loads associated with the $6 g$ gust, the optimizer fixes $n_{j}$ to 2 for both $d_{1}=0.135 c$ and $d_{1}=0.15 c$ at the different constraint cases. The ratio of bending to axial stiffness $\left(K_{z} / K_{x}\right)$ decreases from 8.30 with Case 1 to 1.10 with Case 3 for $d_{1}=0.135 c$. On the other hand, the ratio $\left(K_{z} / K_{x}\right)$ decreases from 10.2 with Constraint 1 to 0.82 with Constraint 3 for $d_{1}=0.15 c$.

Fig. 11 shows the variation of the axial stiffness for the different aspect ratio cases. The curves in Fig. 11 are achieved by using exponential fitting to the data points in Tables 6 and 7. The aim of the

\begin{tabular}{|c|c|c|c|c|c|c|}
\hline \multirow[b]{2}{*}{ Parameter } & \multicolumn{2}{|c|}{ Case 1} & \multicolumn{2}{|c|}{ Case 2} & \multicolumn{2}{|c|}{ Case 3} \\
\hline & $d_{1}=0.135 c$ & $d_{1}=0.15 c$ & $d_{1}=0.135 c$ & $d_{1}=0.15 c$ & $d_{1}=0.135 c$ & $d_{1}=0.15 c$ \\
\hline$K_{x}(\mathrm{kN} / \mathrm{m})$ & 0.65 & 0.52 & 1.25 & 1.95 & 5.10 & 6.60 \\
\hline$K_{z}(\mathrm{kN} / \mathrm{m})$ & 5.40 & 5.30 & 6.00 & 6.00 & 5.40 & 5.40 \\
\hline$K_{z} / K_{x}$ & 8.30 & 10.2 & 4.80 & 3.10 & 1.10 & 0.82 \\
\hline$m_{p}(\mathrm{~kg})$ & 14 & 14 & 14.5 & 15 & 18 & 20 \\
\hline$n_{j}$ & 2 & 2 & 2 & 2 & 2 & 2 \\
\hline$l_{1_{\text {tot }}}^{J}(\mathrm{~m})$ & 0.29 & 0.30 & 0.29 & 0.29 & 0.28 & 0.27 \\
\hline$l_{2_{\text {tot }}}^{\text {tot }}(\mathrm{m})$ & 0.56 & 0.54 & 0.52 & 0.45 & 0.42 & 0.39 \\
\hline$l_{3_{\text {tot }}}^{\text {tot }}(\mathrm{m})$ & 0.35 & 0.36 & 0.39 & 0.46 & 0.50 & 0.54 \\
\hline$l_{e}^{\text {tot }}(\mathrm{m})$ & 0.0075 & 0.0070 & 0.008 & 0.0080 & 0.017 & 0.0195 \\
\hline$d_{3}(\mathrm{~m})$ & 0.058 & 0.056 & 0.090 & 0.127 & 0.074 & 0.077 \\
\hline$t_{1}\left(\times 10^{-3} \mathrm{~m}\right)$ & 2.20 & 1.85 & 1.90 & 1.50 & 1.30 & 1.70 \\
\hline$t_{3}\left(\times 10^{-3} \mathrm{~m}\right)$ & 3.00 & 3.00 & 3.00 & 3.00 & 3.00 & 3.00 \\
\hline$\omega$ (degrees) & 26.0 & 31.0 & 25.0 & 26.0 & 25.0 & 26.0 \\
\hline
\end{tabular}
fitted curves is not to show the actual values but to show the trend of the axial stiffness with the elastomer aspect ratio. With Case 1, the

Table 6. Minimum Axial Stiffness for Different Constraint Cases 


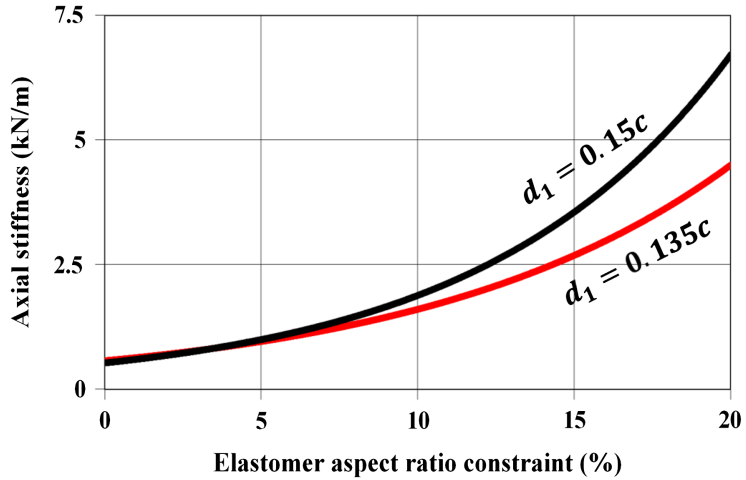

Fig. 11. Variation of axial stiffness for different aspect ratio constraint cases of the elastomeric material

Table 7. Shear Strain of the Elastomer for the Optimum Solutions

\begin{tabular}{lccccccc}
\hline & \multicolumn{3}{c}{$d_{1}=0.135 c$} & & \multicolumn{3}{c}{$d_{1}=0.15 c$} \\
\cline { 2 - 4 } \cline { 7 - 8 } Parameter & $\mathrm{C} 1$ & $\mathrm{C} 2$ & $\mathrm{C} 3$ & & $\mathrm{C} 1$ & $\mathrm{C} 2$ & $\mathrm{C} 3$ \\
\hline Shear strain $(\gamma)(\mathrm{rad})$ & 0.94 & 1.03 & 0.98 & & 0.87 & 1.05 & 0.93 \\
\hline
\end{tabular}

Note: $\mathrm{C} 1=$ Case $1 ; \mathrm{C} 2=$ Case $2 ; \mathrm{C} 3=$ Case 3 .

Table 8. Tip Deflections for the $1 g$ and $6 g$ Loading Scenarios

\begin{tabular}{lcccccccc}
\hline & \multicolumn{3}{c}{$d_{1}=0.135 c$} & & \multicolumn{3}{c}{$d_{1}=0.15 c$} \\
\cline { 2 - 4 } Parameter & $\mathrm{C} 1$ & $\mathrm{C} 2$ & $\mathrm{C} 3$ & & $\mathrm{C} 1$ & $\mathrm{C} 2$ & $\mathrm{C} 3$ \\
\hline$\delta z$ at $1 g(\mathrm{~m})$ & 0.057 & 0.053 & 0.034 & & 0.06 & 0.052 & 0.059 \\
$z z$ at $6 g$ with $\mathrm{SF}=1.5(\mathrm{~m})$ & 0.570 & 0.530 & 0.330 & & 0.59 & 0.520 & 0.580 \\
\hline
\end{tabular}

Note: $\mathrm{C} 1=$ Case $1 ; \mathrm{C} 2=$ Case $2 ; \mathrm{C} 3=$ Case 3 .

axial stiffness associated with $d_{1}=0.15 c$ is $25 \%$ lower than that associated with $d_{1}=0.135 c$. However, when there is a constraint on the elastomer's aspect ratio (10 and 20\%), the axial stiffness associated with $d_{1}=0.15 \mathrm{c}$ is higher than that associated with $d_{1}=0.135 c$. It should be noted that for both $d_{1}=0.135 c$ and $d_{1}=0.15 c$, the GA tends to shift $l_{1_{\text {tot }}}$ to its upper limit $\left(0.25 l_{p}\right)$. On the other hand, $l_{2_{\text {tot }}}$ decreases with an increasing aspect ratio constraint, while $l_{3_{\text {tot }}}$ increases with an increasing value of the aspect ratio constraint.

The shear strains in the elastomeric material associated with each value of $d_{1}$ at the aspect ratio cases are listed in Table 7 . The shear strains listed in Table 8 are very large but well below the allowable shear strain of polyurethane elastomer. However, at such large strains, the stress-strain curve becomes nonlinear and this is not captured in this analysis. Future work will address geometric and material nonlinearity associated with the compliant spar.

Furthermore, the variation of the von Mises stresses on the following optimum solutions is shown in Figs. 12 and 13:

- $d_{1}=0.135 c$ with Case 1 ; and

- $d_{1}=0.15 c$ with Case 1 .

Figs. 12 and 13 show the stress variations for the optimum solutions at the normal $1 g$ loading and at the ultimate $6 g$ loading scenarios.

It should be noted that the front spar is subject to higher bending loads than the rear spar due to the equivalent torque applied, since the spars resist the equivalent torque mainly by bending. The front spar bends up due to the torque which adds up to the bending loads
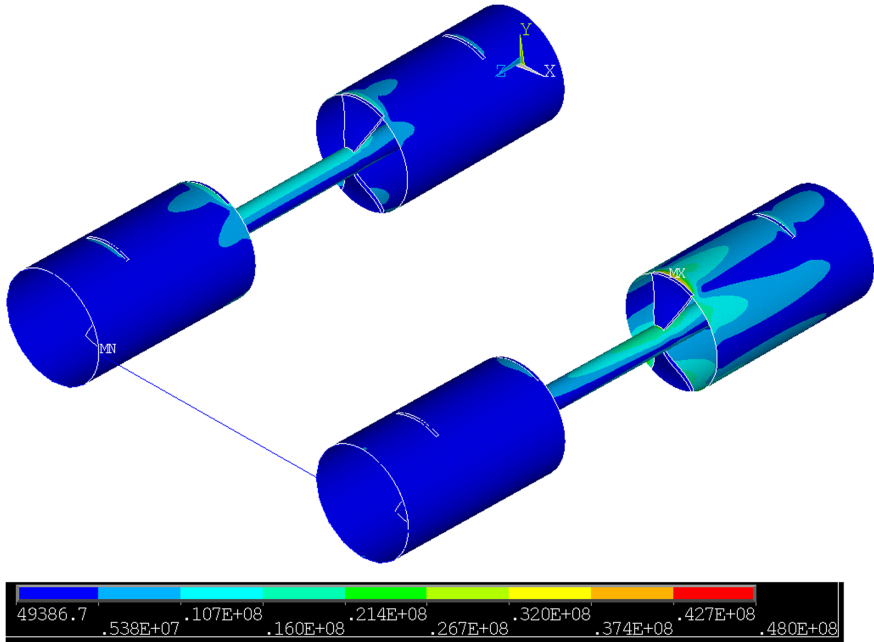

(a)
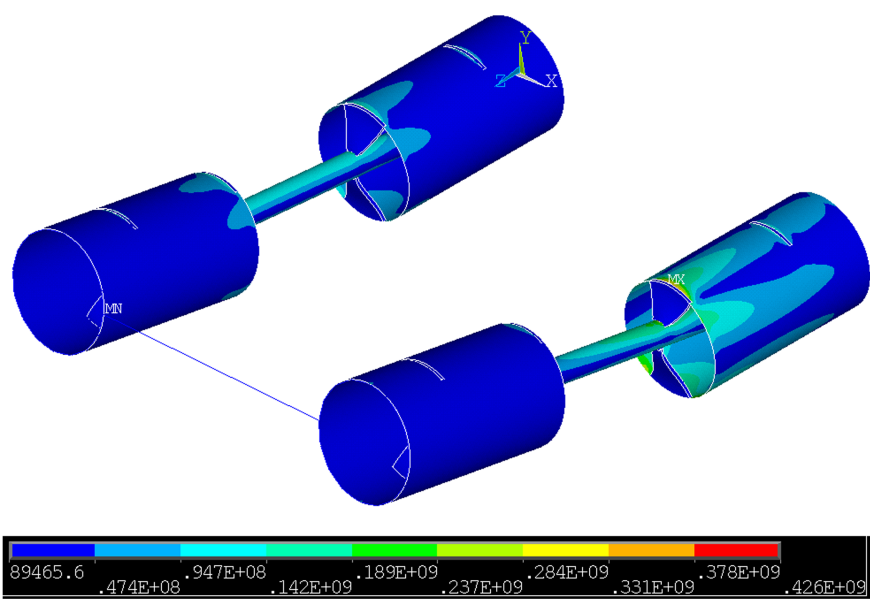

(b)

Fig. 12. Variation of the von Mises stresses for $d_{1}=0.135 c$ with Case 1: (a) at $1 g$ loading; (b) at $6 g$ loading with $\mathrm{SF}=1.5$

due to the equivalent shear force, while the rear spar bends down due to the torque which subtracts from the bending loads due to the equivalent shear force. This results in the front spar carrying higher bending loads and hence, it is subject to higher stresses. This is obvious in Figs. 12 and 13, where the front spar has higher von Mises stresses especially at the interface between the elastomer and the AL tubes close to the root of the partition (clamped). The interface region is highly loaded as the bending loads are transferred from the small AL tube to the large tube through the elastomer. The contact area between the elastomer and Element 1 is relatively small, resulting in high stress concentrations.

Figs. 12 and 13 show that increasing $d_{1}$ from 13.5 to $15 \%$ of the chord reduces the von Mises stresses in the large AL tube (Element 1) and at the interface between the tubes and the elastomeric material.

The tip deflections of the optimum solutions at $6 g$ with a $\mathrm{SF}=$ 1.5 and at $1 g$ (without SF) with the different constraints are listed in Table 8 . The ratio of the aerodynamic loads at $6 g$ (with a SF $=1.5$ ) to those at $1 g$ is 9 , whereas the ratio of their corresponding deflections (listed in Table 8) is about 10. This amplification is mainly due to the self-weight of the compliant spars. It should be noted that at $1 g$ loading, the partition's displacement in bending of the proposed concept is about $15 \%$ more than the conventional wing concept. However, when the impact of the morphing skin (honeycomb+ 


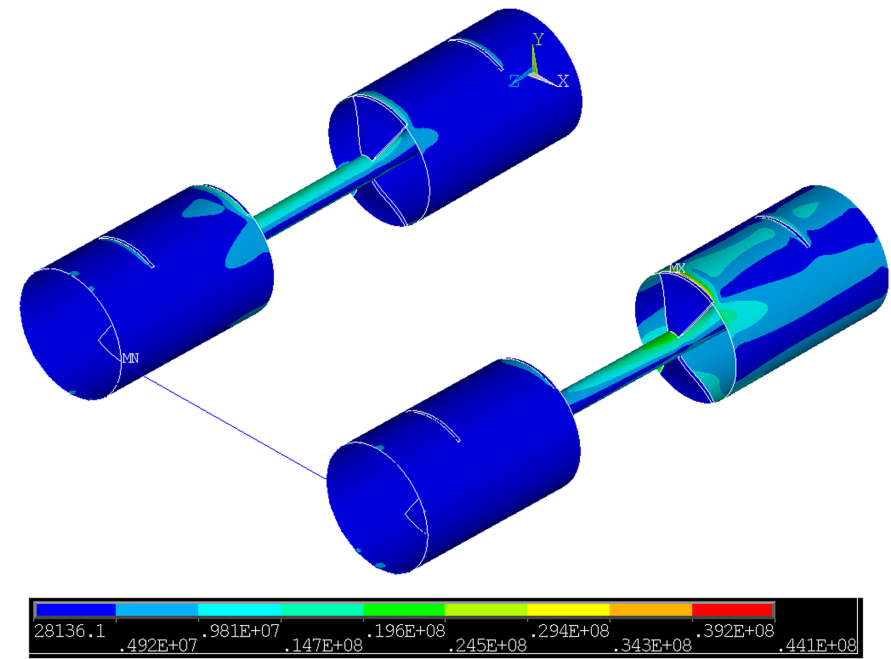

(a)

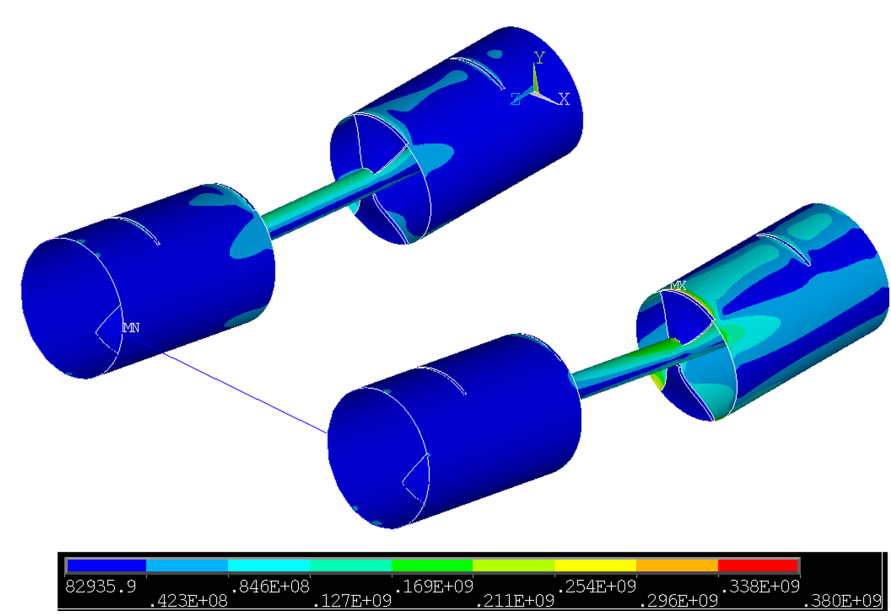

(b)

Fig. 13. Variation of the von Mises stresses for $d_{1}=0.15 c$ with Case 1: (a) at $1 g$ loading; (b) at $6 g$ loading with $\mathrm{SF}=1.5$

elastomeric skin) is considered, the bending stiffness increases and the difference in bending deformation drops from 15 to $10 \%$.

\section{Actuation Forces and Power}

Since the analysis performed here is linear, the actuation forces can be directly extracted from the axial stiffness for a given axial displacement. The total axial displacement $\left(\delta x_{\text {tot }}\right)$ of the partition is $0.264 \mathrm{~m}$. The total actuation force $\left(F_{\text {act }}\right)$ consists of two main contributions, the force to overcome the inertia of the spars and the force to strain them. It should be noted that the contribution of the flexible skin to the actuation force is neglected in this analysis. $F_{\text {act }}$ can be expressed as

$$
F_{\mathrm{act}}=m_{p} a+K_{x} \delta x_{\mathrm{tot}}
$$

where $a=$ acceleration. A uniform accelerated actuation scheme is assumed; hence the acceleration can be expressed as

$$
a=2 \delta x_{\mathrm{tot}} / t_{a}^{2}
$$

where $t_{a}=$ total actuation time. Two cases are considered in this analysis. These correspond to $d_{1}=0.135 c$ with Case 1 and $d_{1}=$ $0.15 c$ with Case 1 . The actuation forces and power are estimated
Table 9. Actuation Requirements for $d_{1}=0.135 c$ with Case 1

\begin{tabular}{lcccccc}
\hline & & & \multicolumn{3}{c}{$F_{\text {act }}(\mathrm{N})$} & \\
\cline { 4 - 6 }$t_{a}(\mathrm{~s})$ & $a\left(\mathrm{~m} / \mathrm{s}^{2}\right)$ & $V_{\max }(\mathrm{m} / \mathrm{s})$ & $m_{p} a$ & $K_{x} \delta x_{\text {tot }}$ & Total & $P_{\max }(\mathrm{W})$ \\
\hline 0.25 & 8.45 & 2.11 & 118 & 172 & 290 & 612 \\
0.5 & 2.11 & 1.06 & 31.0 & 172 & 203 & 215 \\
1.0 & 0.53 & 0.53 & 7.00 & 172 & 179 & 95.0 \\
2.0 & 0.13 & 0.26 & 2.00 & 172 & 174 & 45.0 \\
\hline
\end{tabular}

Table 10. Actuation Requirements for $d_{1}=0.15 c$ with Case 1

\begin{tabular}{lcccccc}
\hline & & & \multicolumn{3}{c}{$F_{\text {act }}(\mathrm{N})$} & \\
\cline { 4 - 6 }$t_{a}(\mathrm{~s})$ & $a\left(\mathrm{~m} / \mathrm{s}^{2}\right)$ & $V_{\max }(\mathrm{m} / \mathrm{s})$ & $m_{p} a$ & $K_{x} \delta x_{\text {tot }}$ & Total & $P_{\max }(\mathrm{W})$ \\
\hline 0.25 & 8.45 & 2.11 & 118 & 137 & 255 & 538 \\
0.5 & 2.11 & 1.06 & 31.0 & 137 & 168 & 178 \\
1.0 & 0.53 & 0.53 & 7.00 & 137 & 144 & 76.0 \\
2.0 & 0.13 & 0.26 & 2.00 & 137 & 138 & 36.0 \\
\hline
\end{tabular}

and presented in Tables 9 and 10, respectively. An $m_{p}$ of $14 \mathrm{~kg}$ is used for the partition with $d_{1}=0.135 c$ and $d_{1}=0.15 c$.

The contribution of the inertial force $\left(m_{p} a\right)$ to the total actuation force drops significantly as $t_{a}$ increases. At $t_{a}=0.25 \mathrm{~s}$, the inertial force is $41 \%$ of the total force and at $t_{a}=1.0 \mathrm{~s}$, the inertial force is only $4 \%$ of the total actuation force and the maximum actuation power $P_{\max }$ is $95 \mathrm{~W}$ for $d_{1}=0.135 c$ with Case 1 . On the other hand, at $t_{a}=0.25 \mathrm{~s}$, the inertial force is $46 \%$ of the total force while at $t_{a}=1.0 \mathrm{~s}$, the inertial force is only $5 \%$ of the total actuation force and the maximum actuation power $P_{\max }$ is $75 \mathrm{~W}$ for $d_{1}=$ $0.15 c$ with Case 1 .

The actuation energy is mainly needed to strain the elastomeric material. The majority of this actuation energy is stored in the form of strain energy in the elastomer. Once the locking mechanism is released, the stored strain energy returns the partition to its original configuration. This is an advantage for the compliant spar in comparison with telescopic spars that require actuation energy to morph and unmorph them. Although the telescopic spar requires actuation energy during extension and retraction, the energy needed is lower than that required for the compliant spar. However, telescopic spars tend to be heavy due to the need for bearings to ease the actuation and to constrain the spar in torsion.

After thorough consideration of the state-of-the-art actuation technologies, electromechanical actuators are selected for the compliant spar concept due to their good performance and low associated weight. The lead angle of the screw is low to provide a self-locking mechanism without the need for continuous power supply to hold in any specific position.

\section{Shear Strain Constraint}

In the previous section, the wingtip partition was designed to have a tip deflection smaller than or equal to $50 \%$ of its length at the ultimate loading scenario ( $6 g$ with a $\mathrm{SF}=1.5$ ). Therefore, the GA optimizer reduced the number of joints to two per spar to maintain sufficient bending stiffness for the different constraints. This resulted in higher shear strains in the elastomer (due to a lower number of joints). In this section, the partition is designed to have a tip deflection smaller than or equal to $10 \%$ of its length at the $1 g$ normal loading scenario. The tip deflections at the ultimate loading scenario are not considered. Constraints on the thicknesses of the AL tubes are added to ensure that they do not fail or undergo permanent deformation when subject to the ultimate loads. The main driver of this section is to reduce the shear strain in the elastomer. 

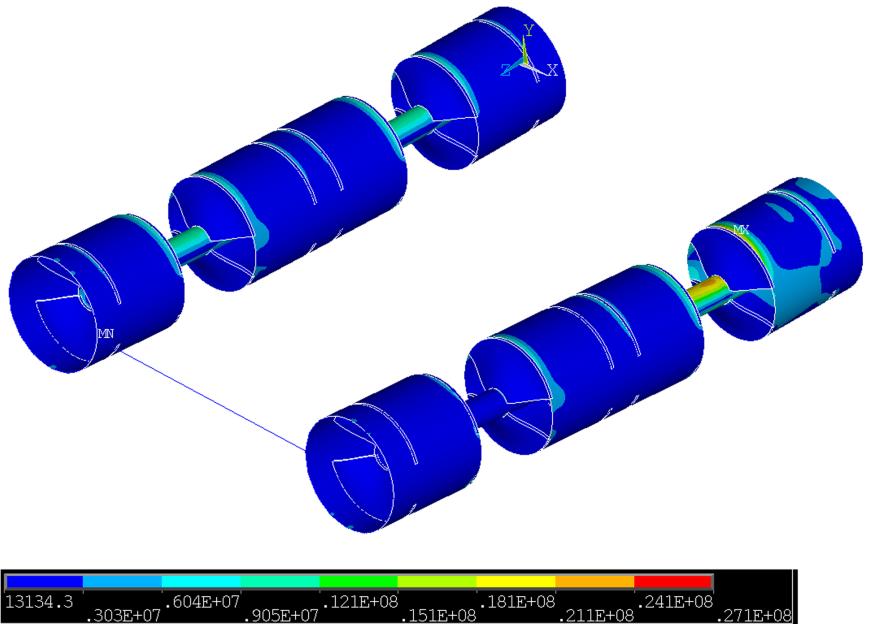

(a)

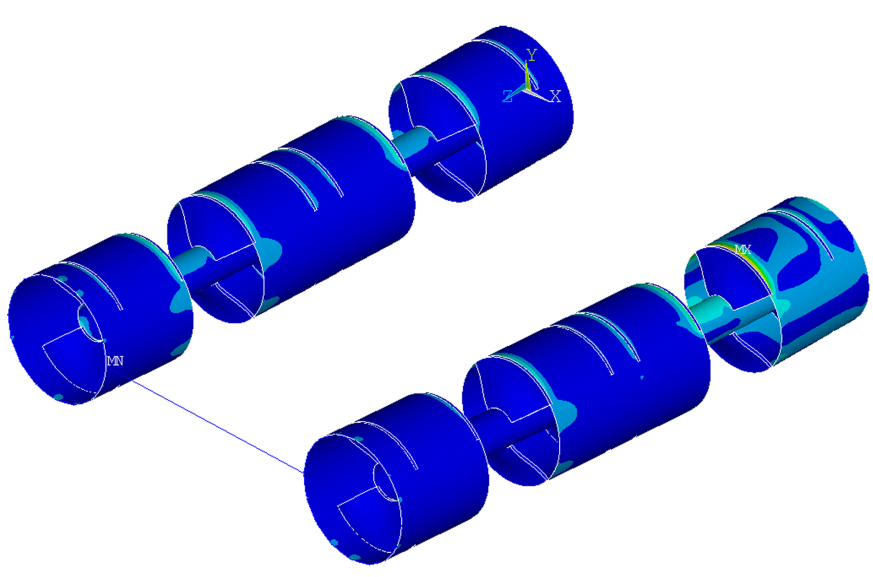

12824.4
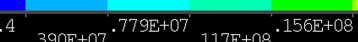

$4 \mathrm{E}+08^{.233 \mathrm{E}+08} \cdot 272 \mathrm{E}+08^{-311 \mathrm{E}+08}$

(b)

Fig. 14. Variation of the von Mises stresses for the optimum solutions at $1 \mathrm{~g}$ with a shear strain constraint: (a) for $d_{1}=0.135 c$; (b) for $d_{1}=0.15 c$

Therefore, a constraint on the shear strain in the elastomer is imposed to ensure that it does not exceed $0.75 \mathrm{rad}$. The analysis is performed for both $d_{1}=0.135 c$ and $d_{1}=0.15 c$ with Case 1 . The optimization problems are summarized in Tables $11\left(d_{1}=\right.$ $0.135 c)$ and $12\left(d_{1}=0.15 c\right)$. The outcomes of the optimization problems are summarized in Table 13. Table 14 shows that shear strain drops from $0.94 \mathrm{rad}$ when the partition is designed for the ultimate loadings (without a shear strain constraint) to $0.63 \mathrm{rad}$

Table 11. Minimized Axial Stiffness for $d_{1}=0.135 c$ at the $1 g$ Flight Condition

\begin{tabular}{lcc}
\hline Objective function & Minimize $K_{x}$ \\
\hline Variables & $n_{j}, l_{1_{\mathrm{tot}}}, l_{2_{\mathrm{tot}}}, l_{e}, d_{3}, t_{1}, t_{3}, \omega, \gamma$ \\
Constraints & $2 \leq n_{j} \leq 6$ & $0.025 l_{2} \leq l_{e} \leq 0.25 l_{2}$ \\
& $0.05 l_{p} \leq l_{1_{\mathrm{tot}}} \leq 0.25 l_{p}$ & $0.03 c \leq d_{3} \leq 0.08 c$ \\
$0.30 l_{p} \leq l_{2_{\mathrm{tot}}} \leq 0.65 l_{p}$ & $25^{\circ} \leq \omega \leq 75^{\circ}$ \\
$l_{1} \geq \delta x_{j}$ & $\gamma \leq 0.75 \mathrm{rad}$ \\
$l_{3} \geq 2 \delta x_{j}$ & $d_{1}=0.135 c$ \\
$\delta z \leq 0.1 l_{p}$ & $\mathrm{RF}_{1} \geq 1$ \\
& $t_{1}=2.20\left(\times 10^{-3} \mathrm{~m}\right)$ & $\mathrm{RF}_{2} \geq 1$ \\
$t_{3}=3.00\left(\times 10^{-3} \mathrm{~m}\right)$ & $\mathrm{RF}_{3} \geq 1$ \\
\hline
\end{tabular}

Table 12. Minimized Axial Stiffness for $d_{1}=0.15 c$ at the $1 g$ Flight Condition

\begin{tabular}{lcc}
\hline Objective function & Minimize $K_{x}$ \\
\hline Variables & $n_{j}, l_{1_{\text {tot }}}, l_{2_{\text {tot }}}, l_{e}, d_{3}, t_{1}, t_{3}, \omega, \gamma$ \\
Constraints & $2 \leq n_{j} \leq 6$ & $0.025 l_{2} \leq l_{e} \leq 0.25 l_{2}$ \\
& $0.05 l_{p} \leq l_{1_{\text {tot }} \leq 0.25 l_{p}}$ & $0.03 c \leq d_{3} \leq 0.08 c$ \\
& $0.30 l_{p} \leq l_{2_{\text {tot }}} \leq 0.65 l_{p}$ & $25^{\circ} \leq \omega \leq 75^{\circ}$ \\
$l_{1} \geq \delta x_{j}$ & $\gamma \leq 0.75 \mathrm{rad}$ \\
$l_{3} \geq 2 \delta x_{j}$ & $d_{1}=0.15 c$ \\
& $\delta z \leq 0.1 l_{p}$ & $\mathrm{RF}_{1} \geq 1$ \\
& $t_{1}=1.85\left(\times 10^{-3} \mathrm{~m}\right)$ & $\mathrm{RF}_{2} \geq 1$ \\
$t_{3}=3.00\left(\times 10^{-3} \mathrm{~m}\right)$ & $\mathrm{RF}_{3} \geq 1$ \\
\hline
\end{tabular}

Table 13. Outcomes of the Optimization Problems

\begin{tabular}{lcc}
\hline & \multicolumn{2}{c}{ Case 1} \\
\cline { 2 - 3 } Parameter & $d_{1}=0.135 c$ & $d_{1}=0.15 c$ \\
\hline$K_{x}(\mathrm{kN} / \mathrm{m})$ & 0.80 & 0.66 \\
$K_{z}(\mathrm{kN} / \mathrm{m})$ & 2.55 & 2.55 \\
$K_{z} / K_{x}$ & 3.20 & 3.90 \\
$m_{p}(\mathrm{~kg})$ & 18 & 20 \\
$n_{j}$ & 4 & 4 \\
$l_{1_{\text {tot }}}(\mathrm{m})$ & 0.73 & 0.73 \\
$l_{2_{\text {tot }}}(\mathrm{m})$ & 0.21 & 0.21 \\
$l_{\text {tot }}(\mathrm{m})$ & 0.26 & 0.26 \\
$l_{e}(\mathrm{~m})$ & 0.007 & 0.007 \\
$d_{3}(\mathrm{~m})$ & 0.070 & 0.090 \\
$\omega(\mathrm{degrees})$ & 68 & 50 \\
$\gamma(\mathrm{rad})$ & 0.63 & 0.61 \\
\hline
\end{tabular}

when it is designed for the $1 g$ normal loading (with shear strain constraint) for $d_{1}=0.135 c$ with Case 1 , and it drops from 0.87 to $0.61 \mathrm{rad}$ for $d_{1}=0.15 \mathrm{c}$ with Case 1 . This is mainly due to the higher $n_{j}$. Increasing $n_{j}$ reduces the shear strain and the axial stiffness but it also reduces significantly the bending stiffness. In order to maintain the bending stiffness, $l_{e}$ and $\omega$ are increased by the optimizer which, in the end, results in a higher axial stiffness when compared to the case of ultimate loads without a shear strain constraint. It should be noted that the values of $l_{1_{\mathrm{tot}}}, l_{2_{\mathrm{tot}}}$, and $l_{3_{\mathrm{tot}}}$ are the same for $d_{1}=0.135 c$ and $d_{1}=0.15 c$. The GA optimizer maximizes the $l_{1_{\text {tot }}}$ to its upper limit to increase the bending stiffness of the partition. The variation of von Mises stresses on the optimal solutions is shown in Fig. 14. The lower shear strains in the elastomer ensure that the assumption of linear elastic behavior is valid.

\section{Structural Mass}

After minimizing the axial stiffness of the partition for different values of $d_{1}$ at different geometric/aspect ratio constraints, the

Table 14. Minimized Structural Mass for $d_{1}=0.135 c$ with Case 2

\begin{tabular}{lcc}
\hline Objective function & Minimize $m_{p}$ \\
\hline Variables & $l_{1_{\text {tot }}, t_{1}, t_{3}}$ \\
Constraints & $0.05 l_{p} \leq l_{1_{\text {tot }}} \leq 0.5 l_{p}$ \\
& $0.10 \leq t_{1} \leq 2.35\left(\times 10^{-3} \mathrm{~m}\right)$ \\
$0.10 \leq t_{3} \leq 3.00$ & $\left(\times 10^{-3} \mathrm{~m}\right)$ \\
$l_{1} \geq \delta x_{j}$ & $\mathrm{RF}_{1} \geq 1$ \\
$l_{3} \geq 2 \delta x_{j}$ & $\mathrm{RF}_{2} \geq 1$ \\
$\delta z \leq 0.5 l_{p}$ & $\mathrm{RF}_{3} \geq 1$ \\
$d_{1}=0.135 c$ & $n_{j}=2$ \\
$l_{e}=0.008 \mathrm{~m}$ & $\omega=25.0^{\circ}$ \\
& $d_{3}=0.090 \mathrm{~m}$ & $l_{l_{\mathrm{tot}}}=0.43 l_{p}$ \\
\hline
\end{tabular}


Table 15. Minimized Structural Mass for $d_{1}=0.15 c$ with Case 2

\begin{tabular}{lcc}
\hline Objective function & Minimize $m_{p}$ \\
\hline Variables & $l_{1_{\text {tot }}, t_{1}, t_{3}}$ \\
Constraints & $0.05 l_{p} \leq l_{1_{\text {tot }}} \leq 0.5 l_{p}$ \\
& $0.10 \leq t_{1} \leq 2.35$ & $\left(\times 10^{-3} \mathrm{~m}\right)$ \\
$0.10 \leq t_{3} \leq 3.00$ & $\left(\times 10^{-3} \mathrm{~m}\right)$ \\
$l_{1} \geq \delta x_{j}$ & $\mathrm{RF}_{1} \geq 1$ \\
$l_{3} \geq 2 \delta x_{j}$ & $\mathrm{RF}_{2} \geq 1$ \\
$\delta z \leq 0.5 l_{p}$ & $\mathrm{RF}_{3} \geq 1$ \\
$d_{1}=0.15 c$ & $n_{j}=2$ \\
& $l_{e}=0.008 \mathrm{~m}$ & $\omega=26.0^{\circ}$ \\
$d_{3}=0.127 \mathrm{~m}$ & $l_{2_{\mathrm{tot}}}=0.375 l_{p}$ \\
\hline
\end{tabular}

Table 16. Minimum Structural Mass of the Partition with Case 2

\begin{tabular}{lcc}
\hline & \multicolumn{2}{c}{ Case 2} \\
\cline { 2 - 3 } Parameter & $d_{1}=0.135 c$ & $d_{1}=0.15 c$ \\
\hline$m_{p}(\mathrm{~kg})$ & 8 & 9 \\
$K_{x}(\mathrm{kN} / \mathrm{m})$ & 1.24 & 1.95 \\
$n_{j}$ & 2 & 2 \\
$l_{1_{\text {tot }}}(\mathrm{m})$ & 0.38 & 0.31 \\
$l_{2_{\text {tot }}}(\mathrm{m})$ & 0.52 & 0.45 \\
$l_{3_{\text {tot }}}(\mathrm{m})$ & 0.30 & 0.44 \\
$l_{e}(\mathrm{~m})$ & 0.008 & 0.008 \\
$d_{3}(\mathrm{~m})$ & 0.090 & 0.127 \\
$t_{1}\left(\times 10^{-3} \mathrm{~m}\right)$ & 0.75 & 0.94 \\
$t_{3}\left(\times 10^{-3} \mathrm{~m}\right)$ & 0.90 & 0.80 \\
$\omega($ degrees $)$ & 25.0 & 26.0 \\
\hline
\end{tabular}

optimum solutions are further optimized to reduce the structural mass without affecting the axial stiffness and violating the bending deformation constraint $(\delta z)$. Only two cases are considered (without the shear strain constraint) in this section. These correspond to $d_{1}=0.135 c$ with Case 2 and $d_{2}=0.15 c$ with Case 2. Tables 14 and 15 summarize the optimization problem for each case. The number of generations for each case is reduced to 50, with 100 individuals per generation, because the number of design variables has decreased in comparison to the axial stiffness optimization. Table 16 summarizes the outcomes of the study. For $d_{1}=0.135 c$, the mass of the partition (only the spars) decreases from 14.5 to $8 \mathrm{~kg}$, which corresponds to a $45 \%$ reduction in the structural mass. On the other hand, for $d_{1}=0.15 c$ the mass decreases from 15 to $9 \mathrm{~kg}$, which corresponds to a $40 \%$ reduction in the structural mass of the partition.

\section{Conclusions}

The compliant spar has shown to be a promising concept capable of delivering the variation in partition span required. High fidelity modeling of the concept was performed using ANSYS 13. Structural optimization studies using a GA optimizer were conducted to maximize the ratio of bending to axial stiffness and to minimize the structural mass of the concept to enhance its feasibility and preserve the benefits of span morphing. The actuation force is mainly required to strain the spar. An actuation power of about $95 \mathrm{~W}$ is required to morph the two compliant spars in $1 \mathrm{~s}$. Sandwich skin panels consisting of elastomeric matrix composite (EMC) covers reinforced by a zero Poisson's ratio cellular core are adopted to maintain the aerodynamic shape of the airfoil. The impact of scaling this concept for different wing sizes and geometries and its impact on stiffness ratio, weight, and actuation will be investigated in future work.

\section{Acknowledgments}

M. I. Friswell acknowledges the support from the European Research Council under the European Union's Seventh Framework Programme (FP/2007-2013) / ERC Grant Agreement n. [247045]. E. I. Saavedra Flores acknowledges the support of the Department of Civil Engineering, University of Santiago, Chile.

\section{References}

Ajaj, R. M., et al. (2012a). "Structural design and modelling of the zigzag wingbox for span morphing wing." 3 rd RAeS Aircraft Structural Design Conf., Royal Aeronautical Society, London, U.K.

Ajaj, R. M., et al. (2013). "The zigzag wingbox for a span morphing wing." Aerosp. Sci. Technol., 28(1), 364-375.

Ajaj, R. M., et al. (2014). "An integrated conceptual design study using span morphing technology." J. Intell. Mater. Syst. Struct., 25(8), 989-1008.

Ajaj, R. M., Friswell, M. I., Saavedra Flores, E. I., Little, O., and Isikveren, A. T. (2012b). "Span morphing: A conceptual design study." 20th AIAA/ ASME/AHS Adaptive Structures Conf., AIAA-2012-1510, Honolulu, HI.

ANSYS 13 [Computer software]. Academic Research, Help System, ANSYS.

Austin, R. (2010). Unmanned aircraft systems: UAV design, development, and deployment, Wiley, West Sussex, U.K.

Bae, J. S., Seigler, T. M., and Inman, D. J. (2005). "Aerodynamic and aeroelastic characteristics of a variable-span morphing wing." J. Aircr., 42(2), 528-534.

Barbarino, S., Bilgen, O., Ajaj, R. M., Friswell, M. I., and Inman, D. J. (2011). "A review of morphing aircraft." J. Intell. Mater. Syst. Struct., 22(9), 823-877.

Blondeau, J., and Pines, D. (2007). "Design and testing of a pneumatic telescopic wing for unmanned aerial vehicles." J. Aircr., 44(4), 1088-1099.

Blondeau, J., Richeson, J., and Pines, D. J. (2003). "Design, development and testing of a morphing aspect ratio wing using an inflatable telescopic spar." 44th AIAA/ASME/ASCE/AHS/ASC Structures, Structural Dynamics and Materials Conf., American Institute of Aeronautics and Astronautics (AIAA), Reston, VA.

Bubert, E. A., Woods, B. K. S., Lee, K., Kothera, C. S., and Wereley, N. M. (2010). "Design and fabrication of a passive 1D morphing aircraft skin." J. Intell. Mater. Syst. Struct., 21(17), 1699-1717.

Bye, D. R., and McClure, P. D. (2007). "Design of a morphing vehicle." 48th AIAA/ASME/ASCE/AHS/ASC Structures, Structural Dynamics, and Materials Conf., AIAA 2007-1728, Honolulu, HI.

Chipperfield, A. J., and Fleming, P. J. (1996). "The Matlab genetic algorithm toolbox." IEE Colloquium on Applied Control Techniques Using Matlab, Digest No.1995/014.

Chipperfield, A. J., Fleming, P. J., and Fonseca, C. M. (1994). "Genetic algorithm tools for control systems engineering." Proc., 1st Int. Conf. Adaptive Computing in Engineering Design and Control, Plymouth Engineering Design Centre, U.K., 128-133.

Groover, M. P. (2010). Fundamentals of modern manufacturing: Materials, processes, and systems, 4th Ed., Wiley, New York.

Ivanco, T. G., Scott, R. C., Love, M. H., Zink, S., and Weisshaar, T. A. (2007). "Validation of the Lockheed Martin morphing concept with wind tunnel testing." 48th AIAA/ASME/ASCE/AHS/ASC Structures, Structural Dynamics, and Materials Conf., AIAA 2007-2235, Honolulu, HI.

Love, M. H., Zink, P. S., Stroud, R. L., Bye, D. R., Rizk, S., and White, D. (2007). "Demonstration of morphing technology through ground and wind tunnel tests." 48th AIAA/ASME/ASCE/AHS/ASC Structures, Structural Dynamics, and Materials Conf., AIAA 2007-1729, Honolulu, HI.

McCormik, B. W. (1995). Aerodynamics, aeronautics and flight mechanics, 2nd Ed., Wiley, New York.

Melin, T. (2000a). "A vortex lattice Matlab implementation for linear aerodynamic wing applications." Master's thesis, Royal Institute of Technology (KTH), Dept. of Aeronautics, Sweden.

Melin, T. (2000b). "User's guide and reference manual for Tornado." Royal Institute of Technology (KTH), Dept. of Aeronautics, Sweden. 
Murray, G., Gandhi, F., and Bakis, C. (2010). "Flexible matrix composite skins for one-dimensional wing morphing." J. Intell. Mater. Syst. Struct., 21(17), 1771-1781.

Neubauer, M., Gunther, G., and Fullhas, K. (2007). "Structural design aspects and criteria for military UAV." RTO-MP-AVT 145 UAV Design Processes and Criteria, EADS Defence and Security, Florence, Italy.

Seigler, T. M., Bae, J. S., and Inman, D. J. (2004). "Flight control of a variable span cruise missile." Proc., 2004 ASME Int. Mechanical Engineering Congress and Exposition, Dynamic Systems and Control, Anaheim, CA, 565-574.
Seigler, T. M., Neal, D. A., Bae, J. S., and Inman, D. J. (2007). "Modeling and flight control of large-scale morphing aircraft." J. Aircr., 44(4), 1077-1087.

Torenbeek, E. (1992). "Development and application of a comprehensive, design-sensitive weight prediction model for wing structures of transport category aircraft." Rep. LR-693, Delft Univ. of Technology, Delft, Netherlands.

Weisshaar, T. A. (2006). "Morphing aircraft technology—New shapes for aircraft design." Multifunctional Structures / Integration of Sensors and Antennas (pp. O1-1-O1-20). Meeting Proc., RTO-MP-AVT-141, RTO, Neuilly-sur-Seine, France. 